\title{
Comparing Algorithms for Reconstructing Digital off-Axis Fresnel Holograms
}

\author{
Michael Liebling \\ Biological Imaging Center, Beckman Institute, California Institute of Technology, \\ Mail Code 139-74, Pasadena, CA 91125, USA \\ Michael Unser \\ Biomedical Imaging Group, Swiss Federal Institute of Technology Lausanne, \\ Bâtiment de Microtechnique, Station 17, CH-1015 Lausanne, Switzerland
}

\begin{abstract}
Three-dimensional information about an object, such as its depth, may be captured and stored digitally in a single, twodimensional, real-valued hologram acquired in an off-axis geometry. Digital reconstruction of the hologram permits the quantitative retrieval of depth data and object position, or allows post-acquisition focusing on selected scenes. Over the past few decades, a number of reconstruction algorithms have been proposed to perform this task in various experimental conditions and for different purposes (metrology, imaging, etc.). Here, we aim at providing guidelines for deciding which algorithm to apply to a given problem. We evaluate reconstruction procedures based on criteria such as reconstruction quality and computational complexity. We propose a simulation procedure of the acquisition process, that allows us to compare a large body of experimental situations and, because the ground truth is known, achieve quantitative comparison.
\end{abstract}

Keywords: Digital holography, Fresnel transform, Fresnelets, phase retrieval, wave-front reconstruction.

\section{INTRODUCTION}

Digital Fresnel holograms ${ }^{1-4}$ acquired in an off-axis geometry are two-dimensional, real-valued digital images that contain information to reconstruct complex-valued wave-fronts. During acquisition, the object of interest is illuminated, (possibly transilluminated) and the reflected (or transmitted) light is propagated to the recording medium, e.g. a solid-state camera. A reference wave, whose propagation direction is at some angle to that of the object wave (see Fig. 1), creates an interference pattern that is recoded,

$$
I(\mathbf{x})=|\Psi(\mathbf{x})+R(\mathbf{x})|^{2}, \text { with } R(\mathbf{x})=A(\mathbf{x}) \exp [i \vartheta(\mathbf{x})]
$$

where the phase of the reference wave $\vartheta(\mathbf{x})$ is assumed to be known and $\mathbf{x}=(x, y)$. If the reference is a plane wave, it can be written, in the acquisition plane, as

$$
R(\mathbf{x})=A(\mathbf{x}) \exp \left[i\left(k_{x} x+k_{y} y\right)\right]
$$

where $k_{x}$ and $k_{y}$ are the components of the wave vector in the acquisition plane.

The wave-front $\Psi(\mathbf{x})$ in the acquisition plane is related to the wave-front in the vicinity of the object of interest, $\psi(\mathbf{x})$ by the following equation, known as the Fresnel transform

$$
\Psi(\mathbf{x})=\frac{\mathrm{e}^{i k_{\lambda} d}}{i \lambda d} \iint \psi(\xi, \eta) \exp \left\{\frac{i \pi}{\lambda d}\left[(\xi-x)^{2}+(\eta-y)^{2}\right]\right\} \mathrm{d} \xi \mathrm{d} \eta
$$

where $\lambda$ is the wavelength, $k_{\lambda}=2 \pi / \lambda$ is the wavenumber, and $d$ is the distance between the object and the acquisition plane.

Unlike other arrangements for acquiring digital holograms, the off-axis geometry only requires the acquisition of a single image to record all the information to subsequently reconstruct the object wave-front. This approach has clear

M.L.: E-mail: liebling @ caltech.edu, Telephone: +1-626-395-2863 


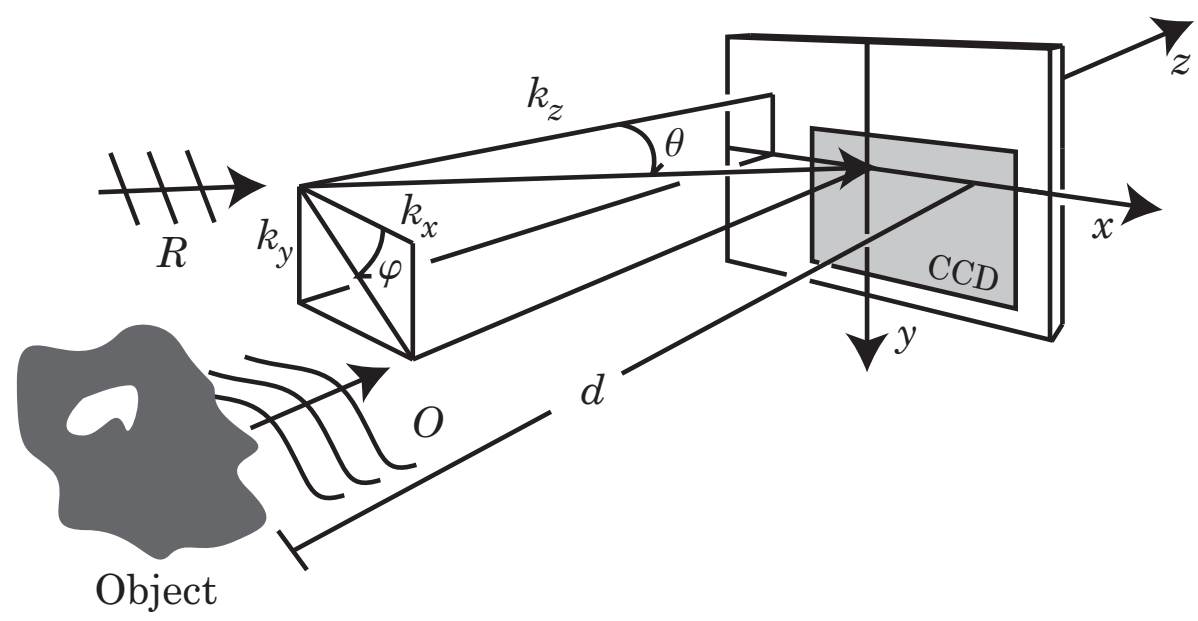

Figure 1. Setup for lensless digital Fresnel off-axis holography.

advantages over methods that require several interferograms to retrieve a complex wave when acquiring time-series, but the increase in temporal resolution comes at the cost of a loss in spatial resolution. Indeed, the presence of fringes reduces the lateral resolution of the reconstructed image.

Many approaches and algorithms for hologram reconstruction have been proposed in recent years. Depending on the parameters of the optical arrangement, they have advantages and disadvantages. Some are suitable for all optical setups while others may only be used in specific situations. We present a qualitative and quantitative approach to the evaluation and comparison of algorithms for the reconstruction of digital off-axis Fresnel holograms. Our aim here is to draw a more complete picture of the relative performances of four algorithms in various optical configurations. The methods are: 1 . reference wave simulation via numerical Fresnel transform, 2. Fourier filtering, 3. non-linear Fresnelet approximation, and 4. a local least-squares wave retrieval procedure. The presented results should facilitate the task of choosing an appropriate algorithm. The comparison is based on the evaluation of the reconstructions from computer generated holograms against a gold standard. Although an evaluation based on synthetic data possibly neglects some aspects present in true experimental conditions, it has the invaluable advantage of giving insight into the performances of the algorithms on a quantitative basis. The situations we present in the following sections are not meant to constitute an exhaustive set of all possible parameter combinations, but it should be possible to apply the methodology to other situations.

There have been only few attempts to quantitatively compare and evaluate the performance of different digital hologram reconstruction algorithms in an extended number of situations. Most validation tests have been carried out on experimental data for which the true gold standard is difficult to access. One approach, proposed by Cuche et al., ${ }^{5,6}$ is to compare the reconstructed phase (respectively, the profile) of a USAF test target to the height measured by scanning a contact-stylus probe profilometer over the same sample. Repeating such an experimental procedure in a large number of configurations would be of highest interest, mainly because it takes into account the whole optical system. However, the time required by such a study would most likely be prohibitive. What we propose here instead is to evaluate the algorithms at hand on a quantitative basis, by restricting the number of influent parameters and by using an exact gold standard. This is made possible by simulating the acquisition process. Even if this does not replace experimental validation, it gives precious insight on the respective impact of each single parameter on the overall performance of the different algorithms. Comparisons of some of the digital versions of the Fresnel transform, ${ }^{3,4,7-12}$ a central part for the reconstruction procedure, have been carried out by Kreis et al.. ${ }^{13}$ In the field of phase retrieval and optical wave-front reconstruction, several comparisons have been proposed. ${ }^{14,15}$

This paper is organized as follows. In Section 2 we present the evaluation methodology and review a list of parameters that influence the acquisition and reconstruction of digital holograms. In Section 3 we briefly outline the algorithms to be compared. In Section 4 we present quantitative evaluation measures for wavefront comparison. In Sections 5 and 6 we present and discuss the results of the comparisons in the case of a lensless setup, synthesize the gathered findings and give an outlook on future work. 


\section{PARAMETERS OF INFLUENCE}

Our evaluation methodology allows for the inclusion of various aspects of the experimental acquisition procedure. In particular, parameters linked to the following components could be taken into account: light source (spatial and temporal coherence, wavelength, intensity profile), optical setup [parasitic reflections, discrepancy from ideal model (nonideal system response, finite apertures, etc.)], object wave (object-hologram distance, size of objects), reference wave (angle to object wave, planarity, intensity ratio to object wave intensity, profile), acquisition device (sampling step, spatial extent, linearity of response, quantization, noise, corrupt regions).

Here, we restrict our study to the influence of the object-hologram distance and the angle between the object and reference wave on the reconstruction quality. In particular, we won't discuss the different implementations of the Fresnel transform per se, although it is central part of to the reconstruction process. For all reconstructions and simulations, we relied on a Fresnelet-based algorithm developed elsewhere. ${ }^{8}$

\section{CONSIDERED ALGORITHMS}

\subsection{Plain Fresnel Transform}

This technique consists in simulating the physical reconstruction process ${ }^{1,3,4,13,16}$ in which the hologram is illuminated by a replica of the reference wave. The latter is diffracted and the three diffraction orders, the image $(+1)$, the twin-image $(-1)$ and the zero order (0) are spatially separated in the object plane. This process may be carried out numerically using a discrete version of the Fresnel transform (3). However, due to the limited angle between object and reference wave imposed by the sampling size of the camera, some overlap between the three orders usually subsists.

\subsection{Fourier Filtering}

In this technique, to avoid the aforementioned overlap problem, the hologram (or, alternatively, the reconstructed wave) is filtered using a band-pass filter to remove the part of the signal that corresponds to the zero and -1 diffraction orders. The filtering is carried out either in the frequency domain or using a digital filter defined in the spatial domain. ${ }^{17-20}$ (See also refs $^{21-23}$ for similar techniques in the general context of interferometry). The techniques mainly vary for the choice of the bandpass filter design. Here, we have chosen to carry out the filtering in the frequency domain using a circular window with smoothed edge, centered around the modulation frequency of the reference wave.

\subsection{Nonlinear Fresnelet Approximation}

It is possible to take advantage of both the separation in space and frequency of the three diffraction orders. In the Fresnelet domain, ${ }^{8}$ the coefficients associated to the contribution of the 0 and -1 orders are separated both spatially and with respect to the frequency bands. It is therefore possible to suppress them by selectively thresholding the Fresnelet coefficients. ${ }^{24}$ Essentially, in regions where the image is not corrupted by the interference terms, no action is taken, while in regions severely corrupted (where the real image and the zero-order overlap) Fresnelet coefficients corresponding to particular frequency bands are thresholded.

\subsection{Least-Squares Estimation (LSE) and Fresnel Transform}

The reconstruction of digital off-axis Fresnel holograms can be decoupled into a phase retrieval problem and a propagation problem. Using this idea, a very flexible reconstruction method has been presented elsewhere. ${ }^{25}$ It consists of two steps: 1. amplitude and phase retrieval in the acquisition plane using a non-linear least-squares estimation (LSE) technique given an a-priori model of the reference wave, and 2. Fresnel propagation up to the correct distance to adjust the focus.

\subsection{Other Methods}

There are several other reconstruction methods that we did not include in this comparison, including statistical methods ${ }^{26}$ or some based on neural nets. ${ }^{27}$ 


\section{COMPARISON METHODOLOGY}

The general methodology for the comparison of the different algorithms consists in: 1. choice of a gold standard wave-front that is representative of an objet, 2. generation (simulation) of a digital hologram, via propagation of the gold standard wave-front, interference with a reference wave, and square-law detection, for various values of the parameters of interest, 3 . reconstruction of the wave-front using the different algorithms, and 4. comparison of the reconstructed and gold standard wave-fronts.

The last step, the comparison of two two-dimensional, complex-valued, sampled functions $f(\mathbf{x})$ and $f^{\prime}(\mathbf{x})$ requires special attention. We first need to recall that phase retrieval algorithms that rely on a single fringe pattern and no a priori knowledge are subject to a sign ambiguity and phase offset inherent to the interference patterns: a general interference pattern given by

$$
|a \exp (i \alpha)+b \exp (i \beta)|^{2}=a^{2}+b^{2}+2 a b \cos (\alpha-\beta),
$$

may equivalently be produced by the phase couples $\alpha^{\prime}, \beta^{\prime}$ :

$$
\begin{aligned}
& \alpha^{\prime}=+\alpha+\delta \\
& \text { and } \\
& \text { or } \alpha^{\prime}=-\alpha+\delta \\
& \text { and } \\
& \begin{array}{l}
\beta^{\prime}=+\beta+\delta, \\
\beta^{\prime}=-\beta+\delta,
\end{array}
\end{aligned}
$$

with $\delta \in \mathbb{R}$. The sign ambiguity may be alleviated by using some a priori knowledge and the phase offset arbitrarily set to zero. Furthermore, only the principal argument of the complex number $Z=a \exp (i \phi) \in \mathbb{C}, a \in \mathbb{R}_{+}, \phi \in \mathbb{R}$ is available and not the phase per se. The phase is said to be rrapped $^{28}$ :

$$
\mathcal{W}\{\phi\}=\arg Z=\phi+2 k \pi, k \in \mathbb{Z}:-\pi<\mathcal{W}\{\phi\} \leq \pi .
$$

We make use of the following congruence property when comparing phases:

$$
\mathcal{W}\left\{\mathcal{W}\left\{\phi_{1}\right\}-\mathcal{W}\left\{\phi_{2}\right\}\right\}=\mathcal{W}\left\{\phi_{1}-\phi_{2}\right\}, \text { if }-\pi<\phi_{1}-\phi_{2}<\pi
$$

Thus, if the difference is sufficiently small, we may evaluate the reconstruction quality without having to unwrap the result.

We define three quality measures for comparing complex-valued images. The first definition of the Signal to noise ratio $(\mathrm{SNR})$ is as follows:

$$
\mathrm{SNR}=10 \log _{10} \frac{\sum_{\mathbf{k}}\left|f_{\mathbf{k}}\right|^{2}}{\sum_{\mathbf{k}}\left|f_{\mathbf{k}}-f_{\mathbf{k}}^{\prime}\right|^{2}}
$$

where $f_{\mathbf{k}}=a_{\mathbf{k}} \mathrm{e}^{i \phi_{\mathbf{k}}} \in \mathbb{C}$ is the gold standard image and $f_{\mathbf{k}}^{\prime}=a_{\mathbf{k}}^{\prime} \mathrm{e}^{i \phi_{\mathbf{k}}^{\prime}} \in \mathbb{C}$ the reconstructed image. Since results in digital holography are usually given in terms of amplitude and phase, we also define the two following quality measures, for the amplitude and the phase, respectively:

$$
\begin{aligned}
\mathrm{SNR}_{\mathrm{amp}} & =10 \log _{10} \frac{\sum_{\mathbf{k}}\left|f_{\mathbf{k}}\right|^{2}}{\sum_{\mathbf{k}}\left(\left|f_{\mathbf{k}}\right|-\left|f_{\mathbf{k}}^{\prime}\right|\right)^{2}} \\
\mathrm{SNR}_{\mathrm{ph}} & =10 \log _{10} \frac{\sum_{\mathbf{k}}\left|\phi_{\mathbf{k}}\right|^{2}}{\sum_{\mathbf{k}}\left(\mathcal{W}\left\{\mathcal{W}\left\{\phi_{\mathbf{k}}\right\}-\mathcal{W}\left\{\phi_{\mathbf{k}}^{\prime}\right\}\right\}\right)^{2}} .
\end{aligned}
$$

The indices $\mathbf{k}$ are taken over a suitable portion of the image. In all subsequent experiments, it covers a centered square whose size is $60 \%$ of the total image width to factor out boundary effects. 


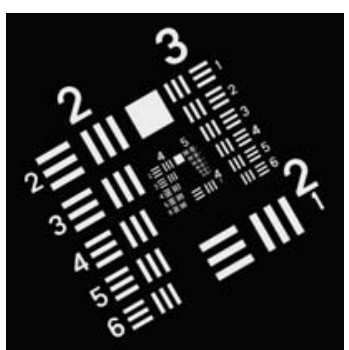

(a)

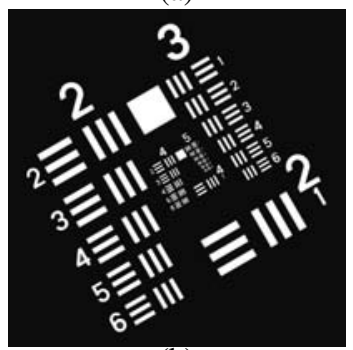

(b)

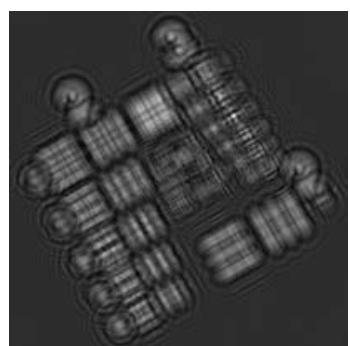

(c)

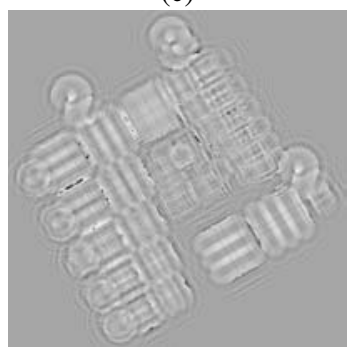

(d)

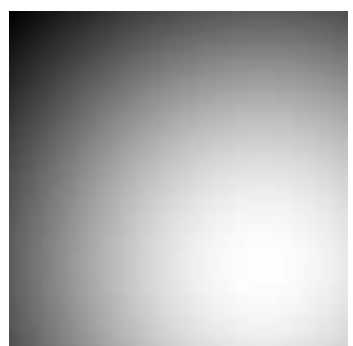

(e)

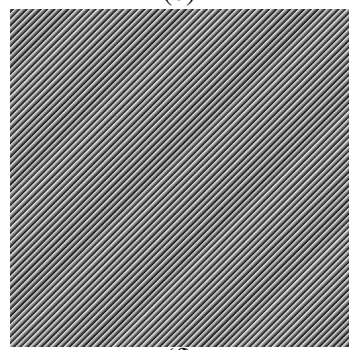

(f)

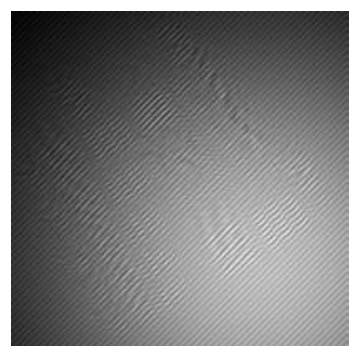

(g)

Figure 2. Gold standard test target object wave. Amplitude $[0.5,1.5]$ (a) and phase $[-0.2 \pi, 0]$ (b) in the vicinity of the object. Amplitude (c) and phase (d) in the CCD plane. Reference wave amplitude (max amplitude: 10) (e) and (wrapped) phase (f). (g) Hologram. Parameters are $T=10 \mu \mathrm{m}, N_{x}=N_{y}=512, \lambda=632.8 \mathrm{~nm}, d=0.05 \mathrm{~m}$ and $K_{x}=K_{y}=-0.6$.

\section{LENSLESS FRESNEL OFF-AXIS HOLOGRAPHY: A COMPARISON}

\subsection{Generation of the Gold Standard}

In this section we examine the four digital hologram reconstruction algorithms in the case of a lensless setup, as shown in Fig. 1. We set the wavelength to $\lambda=632.8 \mathrm{~nm}$. The reference wave vector is $\mathbf{k}_{\lambda}=\left(k_{x}, k_{y}, k_{z}\right)$ and the wavenumber $k_{\lambda}=2 \pi / \lambda=\left(k_{x}^{2}+k_{y}^{2}+k_{z}^{2}\right)^{1 / 2}$. The camera has a sampling step $T=10 \mu \mathrm{m}$ and $N_{x} \times N_{y}=512 \times 512$ pixels. We simulated the propagated wave at a distance $d$ from the object and added a complex reference wave with parameters $K_{x}=k_{x} T$ and $K_{y}=k_{y} T$. We then computed the squared modulus of the resulting field. We sampled the latter on the area corresponding to the camera's support, again with a sampling step $T$. In Fig. 2 we show the steps that lead to the gold standard test target.

\subsection{Distance and Angle}

We now turn to the investigation of the influence on the reconstruction quality of the distance parameter $d$ and the angle between the reference and the object wave. We have considered that the distance between the object and the CCD varies between $0.01 \mathrm{~m}$ and $0.4 \mathrm{~m}$ and generated the corresponding holograms for various angles between the object and reference wave. We have reconstructed the amplitude and phase images using the following algorithms: a plain Fresnel transform, a Fourier filtering followed by Fresnel transform, the Fresnelet approximation and the LSE followed by a Fresnel transform.

In Fig. 3, we show the SNR quality curves for the tested situations. In Figs. 4, 5, and 6 we show the reconstructed amplitudes and phases with the different algorithms for various angles between the reference and the object wave.

We notice that the curves are similar for the overall quality and the amplitude. We see that a larger distance can compensate for a lower angle. When no filtering is used, it is the product $\left(k_{x}+k_{y}\right)^{1 / 2} \lambda d$ which is crucial: the reconstruction is uncorrupted by the zero-order term only if this value is well above the object's support.

One can see that the Fresnelet approximation gives better results (amplitude reconstructions) when a compromise between space and frequency filtering is achievable. This corresponds to regimes where the product $d \lambda\left(k_{x}^{2}+k_{y}^{2}\right)^{1 / 2} /(2 \pi)$ is larger than zero (the different orders do completely overlap) but smaller than the support of the image (the different orders are completely separated in the space domain). As soon as the different orders are well-separated in space, neither the Fourier filtering nor the Fresnelet approximation bring an advantage over the plain Fresnel transform. When the distance is zero, only the Fourier filtering and the LSE algorithms are capable of retrieving meaningful information. It is 


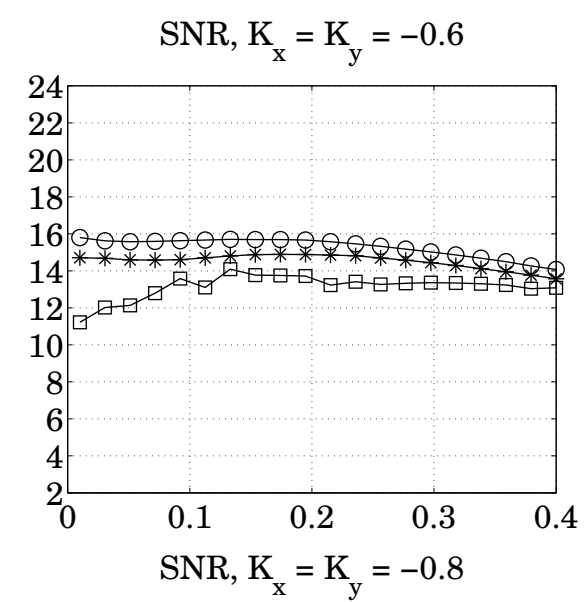

SNR Amplitude, $\mathrm{K}_{\mathrm{x}}=\mathrm{K}_{\mathrm{y}}=-0.6$

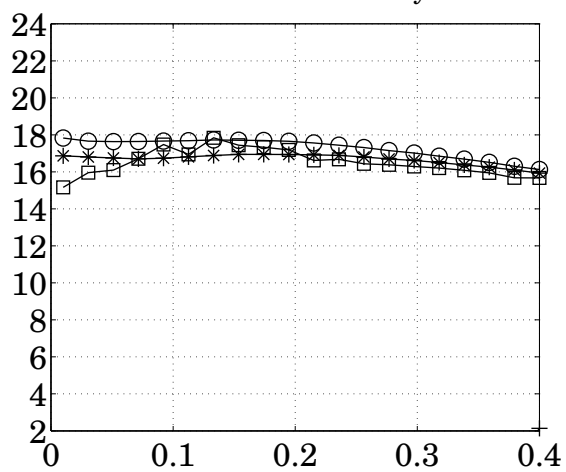

SNR Phase, $\mathrm{K}_{\mathrm{x}}=\mathrm{K}_{\mathrm{y}}=-0.6$

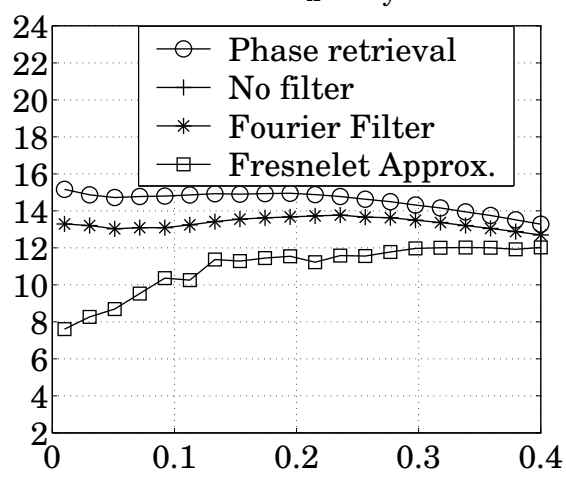

SNR Amplitude, $\mathrm{K}_{\mathrm{x}}=\mathrm{K}_{\mathrm{y}}=-0.8$

SNR Phase, $\mathrm{K}_{\mathrm{x}}=\mathrm{K}_{\mathrm{y}}=-0.8$
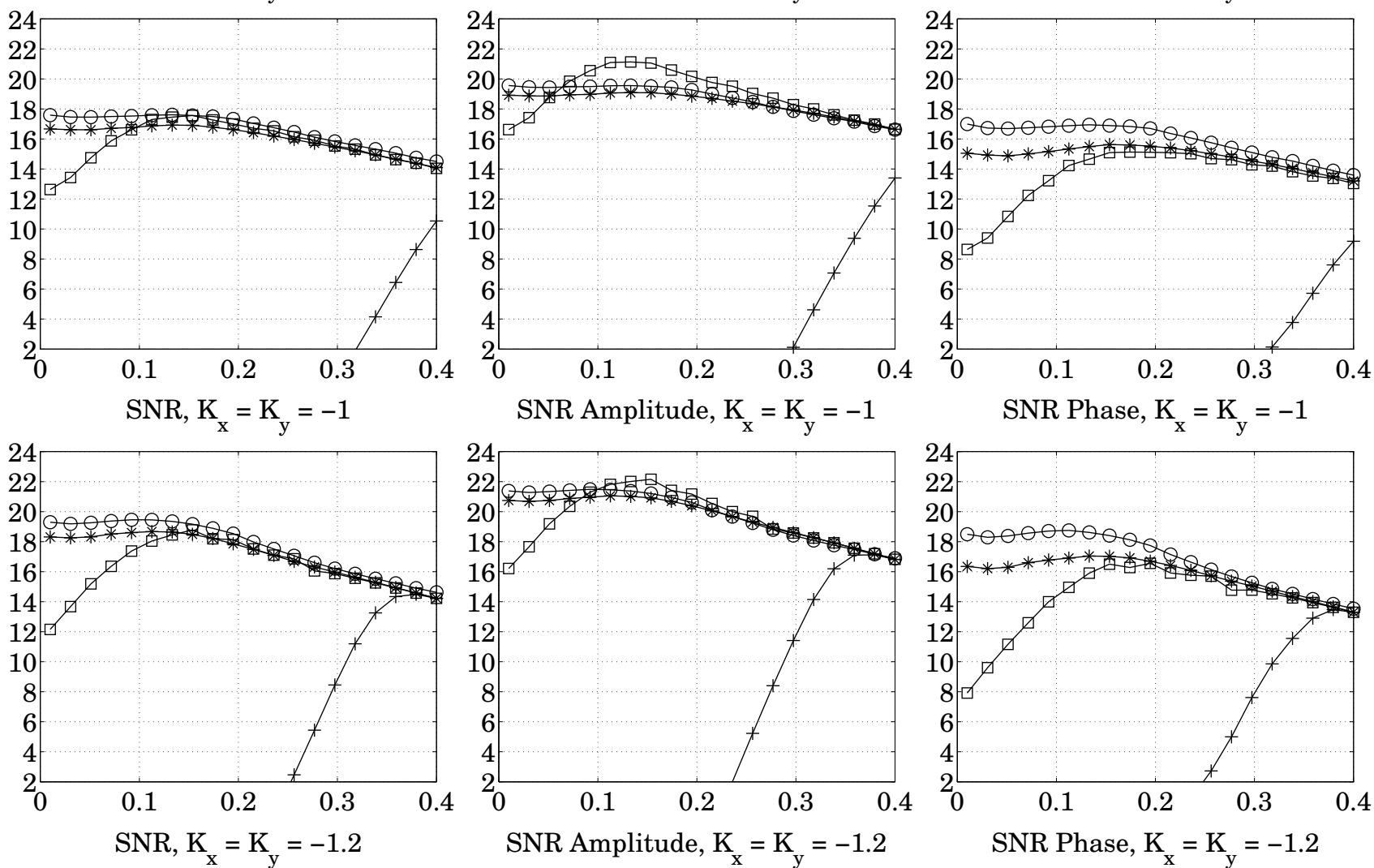

SNR Amplitude, $\mathrm{K}_{\mathrm{x}}=\mathrm{K}_{\mathrm{y}}=-1.2$
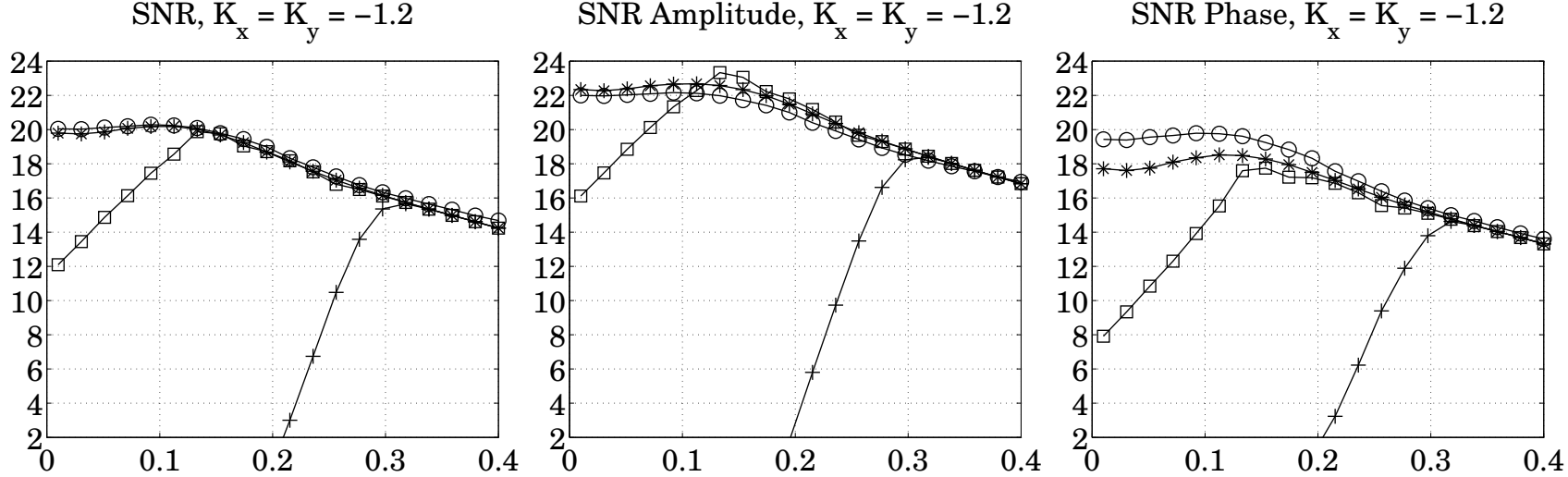

Figure 3. Reconstruction quality $[\mathrm{SNR}]=\mathrm{dB}$ as a function of distance $[d]=\mathrm{m}$. From top to bottom $K_{x}=K_{y}=-0.6,-0.8,-1,-1.2$. 


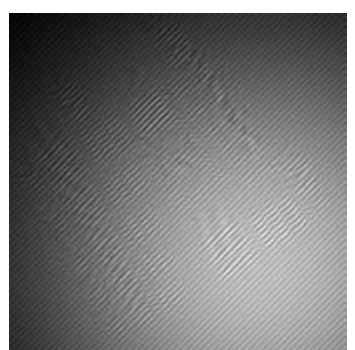

(a)

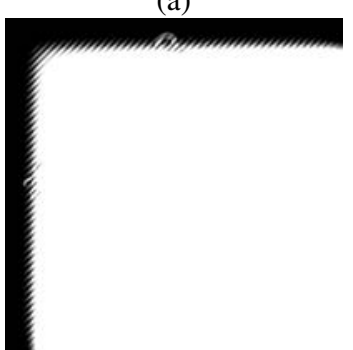

(b)

$\mathrm{SNR}_{\mathrm{amp}}=-19.44$

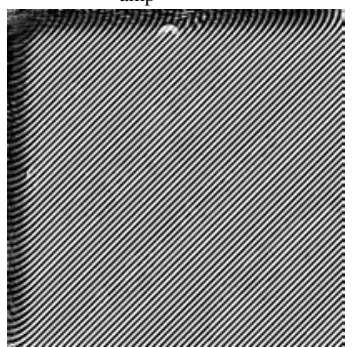

(d)

$\mathrm{SNR}_{\mathrm{ph}}=-9.77$

$\mathrm{SNR}=-20.30$

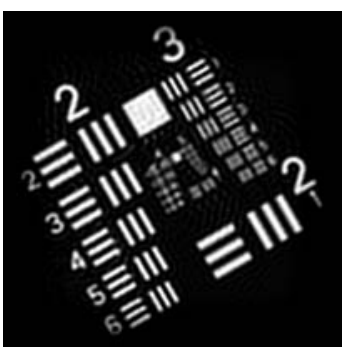

(d)

$\mathrm{SNR}_{\mathrm{amp}}=16.73$

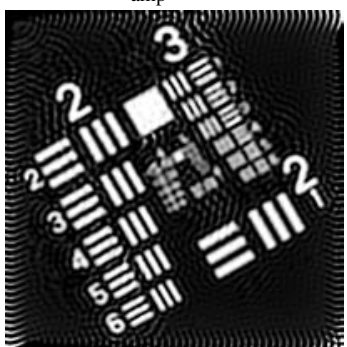

(e)

$\mathrm{SNR}_{\mathrm{ph}}=13.05$

$\mathrm{SNR}=14.60$

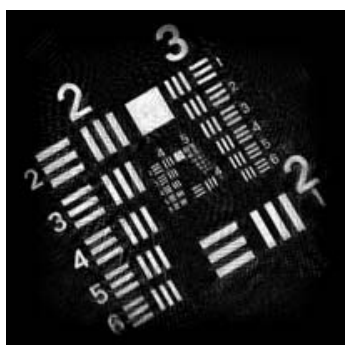

(f)

$\mathrm{SNR}_{\mathrm{amp}}=16.23$

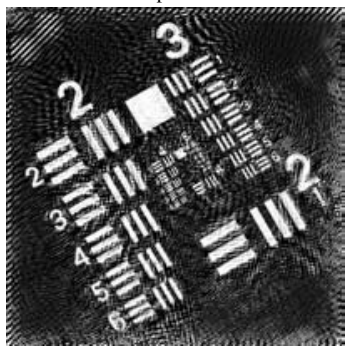

(g)

$\mathrm{SNR}_{\mathrm{ph}}=8.83$

$\mathrm{SNR}=12.27$

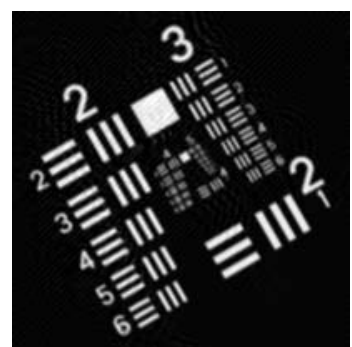

(h)

$\mathrm{SNR}_{\mathrm{amp}}=17.62$

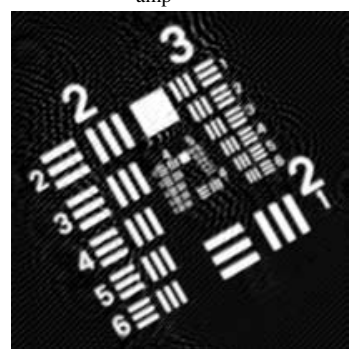

(i)

$\mathrm{SNR}_{\mathrm{ph}}=14.74$

$\mathrm{SNR}=15.58$

Figure 4. Reconstructions (top amplitude, bottom phase) for $d=0.05 \mathrm{~m}$ and $K_{x}=K_{y}=-0.6$. Algorithms from left to right: Plain Fresnel transform, Fourier filtering and Fresnel transform, Fresnelet approximation, LSE and Fresnel transform.

also noteworthy that the LSE method outperforms the other approaches, especially for low $K_{x, y}$, i.e., for low angles. For the higher angles, the Fourier filtering method yields better amplitude results. Also, this is true only if we limit the part of the image that is taken into account for computing the SNR to the center rather than taking the whole field of view. Since the LSE is local, it is less affected by boundary artifacts.

Overall, the reconstruction quality decreases as the distance increases. This is due to the fact that the CCD has a limited support; it therefore misses important information that spreads outside its boundaries. In certain cases, however, a nonzero distance is unavoidable because of space constraints dictated by the optical elements. Generally speaking, we observe that the quality increases as the angle increases. There is of course an upper bound on the maximal angle that is dictated by the CCD's sampling step.

There is no algorithm that is superior to all others in every situation. A method that may seem optimal for a given setup may well become obsolete as soon as a new technology (camera, computer,...) becomes available or economically more attractive. The design of digital holography setups has largely been influenced by the computational limitations of early calculators. The simpler and faster algorithms would be chosen because there was no alternate way to carry out the computations within reasonable time. Such considerations have undoubtedly led to the design of experimental setups optimized for the fastest algorithm. However, methods that were abandoned because they did not meet the speed requirements have not necessarily been reevaluated in the light of the currently available computing power. An algorithm should be adapted to the problem and not the other way around. As a consequence, a flexible algorithm has unquestionable advantages. It should be ready for technological improvements of digital cameras, such as lower sampling steps, larger 


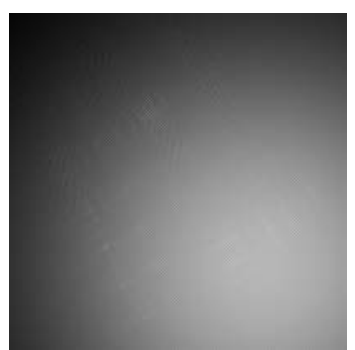

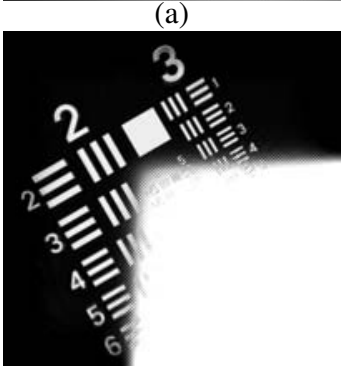

(b)

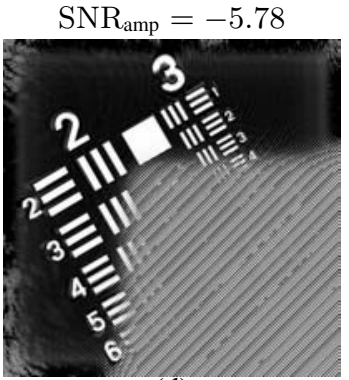

(d)

$\mathrm{SNR}_{\mathrm{ph}}=-3.62$

$\mathrm{SNR}=-7.53$

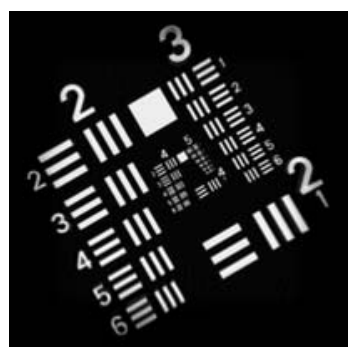

(d)

$\mathrm{SNR}_{\mathrm{amp}}=22.40$

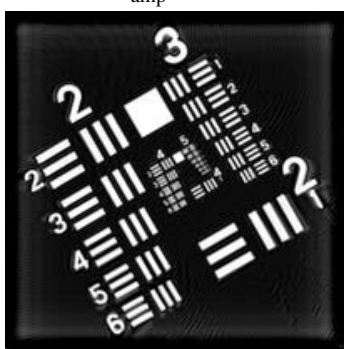

(e)

$\mathrm{SNR}_{\mathrm{ph}}=18.32$

$\mathrm{SNR}=19.76$

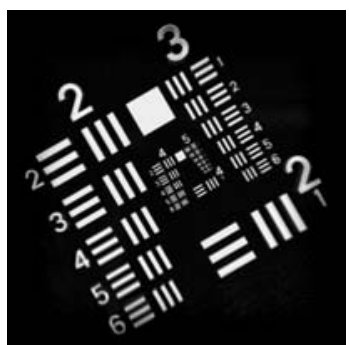

(f)

$\mathrm{SNR}_{\mathrm{amp}}=22.64$

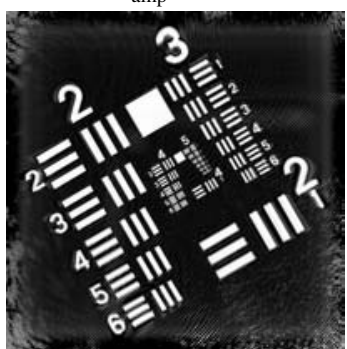

(g)

$\mathrm{SNR}_{\mathrm{ph}}=17.09$

$\mathrm{SNR}=19.30$

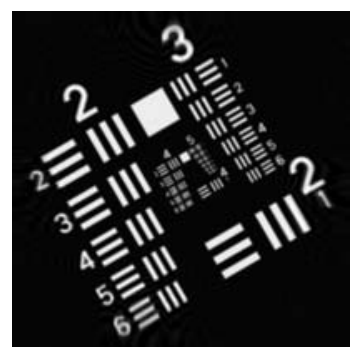

(h)

$\mathrm{SNR}_{\mathrm{amp}}=21.78$

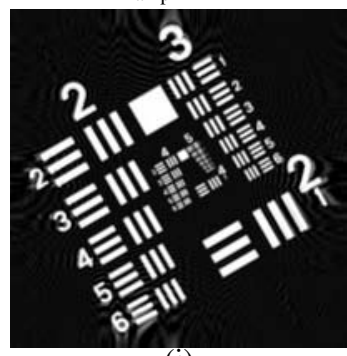

(i)

$\mathrm{SNR}_{\mathrm{ph}}=19.35$

$\mathrm{SNR}=19.86$

Figure 5. Reconstructions (top amplitude, bottom phase) for $d=0.15 \mathrm{~m}$ and $K_{x}=K_{y}=-1.2$. Algorithms from left to right: Plain Fresnel transform, Fourier filtering and Fresnel transform, Fresnelet approximation, LSE and Fresnel transform.

camera size and the increased storage capacity and clock speed to be expected from future computers.

\section{CONCLUSION}

In this paper, we presented a methodology to compare algorithms for the reconstruction of digital Fresnel off-axis holograms. We have compared four digital hologram reconstruction methods in various situations and found that they perform differently depending on the experimental conditions and the measurements of interest (amplitude, phase). The methodology should be suitable for independently examining a wide number of other parameters and methods in a controlled environment.

\section{ACKNOWLEDGMENTS}

The research upon which this paper is based was carried out at the Swiss Federal Institute of Technology Lausanne (EPFL) as part of the doctoral thesis ${ }^{29}$ of the first author.

\section{REFERENCES}

1. J. W. Goodman and R. W. Lawrence, "Digital image formation from electronically detected holograms," Appl. Phys. Lett. 11, pp. 77-79, Aug. 1967. 


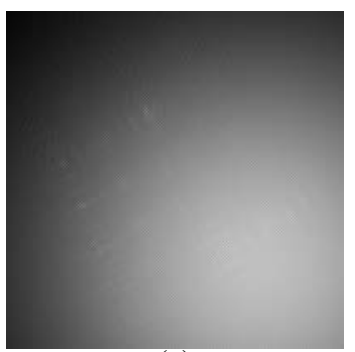

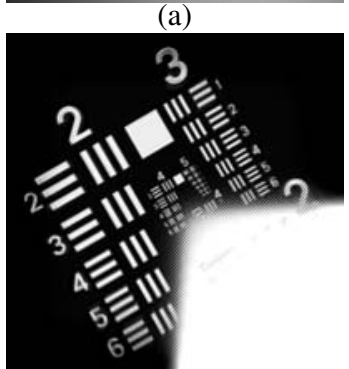

(b)

$\mathrm{SNR}_{\mathrm{amp}}=2.81$

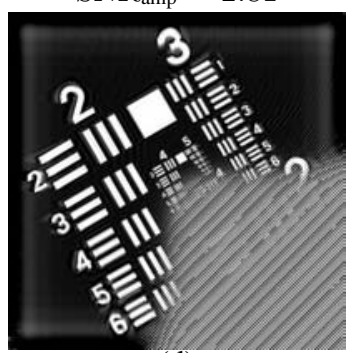

(d)

$\mathrm{SNR}_{\text {ph }}=1.48$

$\mathrm{SNR}=0.35$

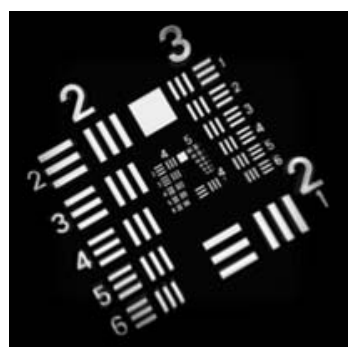

(d)

$\mathrm{SNR}_{\mathrm{amp}}=21.33$

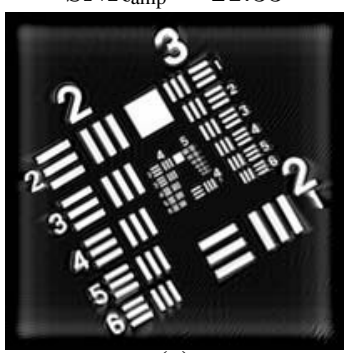

(e)

$\mathrm{SNR}_{\mathrm{ph}}=17.38$

$\mathrm{SNR}=18.53$

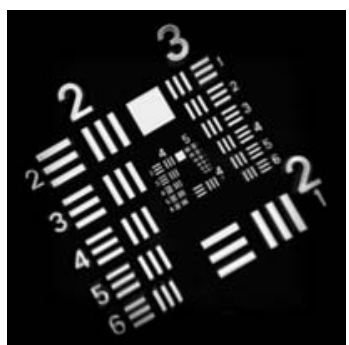

(f)

$\mathrm{SNR}_{\mathrm{amp}}=21.47$

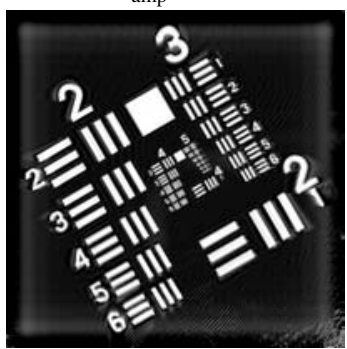

(g)

$\mathrm{SNR}_{\mathrm{ph}}=16.85$

$\mathrm{SNR}=18.40$

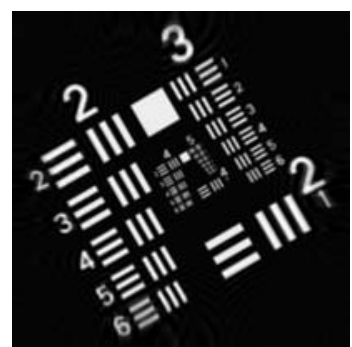

(h)

$\mathrm{SNR}_{\mathrm{amp}}=20.87$

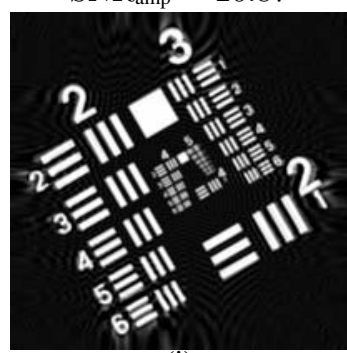

(i)

$\mathrm{SNR}_{\mathrm{ph}}=18.15$

$\mathrm{SNR}=18.84$

Figure 6. Reconstructions (top amplitude, bottom phase) for $d=0.2 \mathrm{~m}$ and $K_{x}=K_{y}=-1.2$. Algorithms from left to right: Plain Fresnel transform, Fourier filtering and Fresnel transform, Fresnelet approximation, LSE and Fresnel transform.

2. M. A. Kronrod, N. S. Merzlyakov, and L. Yaroslavskii, "Reconstruction of a hologram with a computer," Sov. Phys. Tech. Phys. 17, pp. 333-334, Aug. 1972.

3. U. Schnars and W. Jüptner, "Direct recording of holograms by a CCD target and numerical reconstruction," Appl. Opt. 33, pp. 179-181, Jan. 1994.

4. E. Cuche, P. Marquet, and C. Depeursinge, "Simultaneous amplitude-contrast and quantitative phase-contrast microscopy by numerical reconstruction of Fresnel off-axis holograms," Appl. Opt. 38, pp. 6994-7001, Dec. 1999.

5. E. Cuche, F. Bevilacqua, and C. Depeursinge, "Digital holography for quantitative phase-contrast imaging," Opt. Lett. 24, pp. 291-293, Mar. 1999.

6. E. Cuche, Numerical reconstruction of digital holograms : application to phase-contrast imaging and microscopy. $\mathrm{PhD}$ thesis, École Polytechnique Fédérale de Lausanne, Lausanne, 2000.

7. L. P. Yaroslavskii and N. S. Merzlyakov, Methods of Digital Holography, Consultants Bureau, New York, 1980.

8. M. Liebling, T. Blu, and M. Unser, "Fresnelets: new multiresolution wavelet bases for digital holography," IEEE Trans. Image Process. 12, pp. 29-43, Jan. 2003.

9. L. Yaroslavsky and N. Ben-David, "Focal plane invariant algorithm for digital reconstruction of holograms recorded in the near diffraction zone," in Optical Measurement Systems For Industrial Inspection III, W. Osten, K. Creath, and M. Kujawinska, eds., 5144, pp. 142-149, (Munich, Germany), June 22-25, 2003. 
10. P. Ferraro, G. Coppola, D. Alfieri, S. De Nicola, A. Finizio, and G. Pierattini, "Controlling images parameters in the reconstruction process of digital holograms," IEEE Journal of Selected Topics in Quantum Electronics 10, pp. 829839, Jul.-Aug. 2004.

11. B. M. Hennelly and J. T. Sheridan, "Generalizing, optimizing, and inventing numerical algorithms for the fractional Fourier, Fresnel, and linear canonical transforms," J. Opt. Soc. Am. A 22, pp. 917-927, May 2005.

12. B. M. Hennelly and J. T. Sheridan, "Fast numerical algorithm for the linear canonical transform," J. Opt. Soc. Am. A 22, pp. 928-937, May 2005.

13. T. M. Kreis, M. Adams, and W. P. O. Jüptner, "Methods of digital holography: A comparison," in Optical Inspection and Micromeasurements II, C. Gorecki, ed., 3098, pp. 224-233, Proc. SPIE, 1997.

14. J. R. Fienup, “Phase retrieval algorithms: a comparison,” Appl. Opt. 21, pp. 2758-2769, Aug. 1982.

15. D. R. Luke, J. V. Burke, and R. G. Lyon, "Optical wavefront reconstruction: theory and numerical methods," SIAM Review 44, pp. 169-224, June 2002.

16. Q. S. Ru, T. Honda, J. Tsujiuchi, and N. Ohyama, "Fringe analysis by using 2-d Fresnel transform," Optics Communications 66, pp. 21-24, Apr. 1988.

17. T. Kreis, "Digital holographic interference-phase measurement using the Fourier-transform method," J. Opt. Soc. Am. A 3, pp. 847-855, June 1986.

18. T. Kreis and W. P. O. Jüptner, "Suppression of the dc term in digital holography," Opt. Eng. 36, pp. 2357-2360, Aug. 1997.

19. E. Cuche, P. Marquet, and C. Depeursinge, "Spatial filtering for zero-order and twin-image elimination in digital off-axis holography," Appl. Opt. 39, pp. 4070-4075, Aug. 2000.

20. C. Liu, Y. Li, X. Cheng, Z. Liu, F. Bo, and J. Zhu, "Elimination of zero-order diffraction in digital holography," Opt. Eng. 41, pp. 2434-2437, Oct. 2002.

21. M. Takeda, H. Ina, and S. Kobayashi, "Fourier-transform method of fringe-pattern analysis for computer-based topography and interferometry," J. Opt. Soc. Amer. 72, pp. 156-160, Jan. 1982.

22. L. Mertz, "Real-time fringe-pattern analysis," Appl. Opt. 22, pp. 1535-1539, May 1983.

23. C. Roddier and F. Roddier, "Interferogram analysis using Fourier transform techniques," Appl. Opt. 26, pp. 16681673, May 1987.

24. M. Liebling, T. Blu, and M. Unser, "Non-linear Fresnelet approximation for interference term suppression in digital holography," in Wavelets: Applications in Signal and Image Processing X, M. A. Unser, A. Aldroubi, and A. F. Laine, eds., 5207, pp. 553-559, Proc. SPIE, (San Diego CA, USA), August 3-8, 2003.

25. M. Liebling, T. Blu, and M. Unser, "Complex-wave retrieval from a single off-axis hologram," J. Opt. Soc. Am. A 21, pp. 367-377, Mar. 2004.

26. S. Sotthivirat and J. A. Fessler, "Penalized-likelihood image reconstruction for digital holography," J. Opt. Soc. Am. A 21, pp. 737-750, May 2004.

27. R. R. Meyer and E. Heindl, "Reconstruction of off-axis electron holograms using a neural net," J. Microsc.-Oxf. 191, pp. 52-59, July 1998.

28. D. C. Ghiglia and M. D. Pritt, Two-dimensional phase unwrapping, Wiley, New York, 1998.

29. M. Liebling, On Fresnelets, Interference Fringes, and Digital Holography. PhD thesis, Swiss Federal Institute of Technology Lausanne (EPFL), April 21, 2004. EPFL Thesis no. 2977 (2004), 151 p. 